\title{
Features of stable diffuse arcs observed by means of auroral tomography
}

\author{
Zh. V. Dashkevich, V. E. Ivanov, and B. Z. Khudukon \\ Polar Geophysical Institute, 14 Fersmana St., 184209 Apatity, Russia
}

Received: 20 June 2006 - Revised: 28 April 2007 - Accepted: 7 May 2007 - Published: 4 June 2007

\begin{abstract}
In this paper we study the spatial distribution of optical volume emission rates and peculiarities of the luminosity intensity within weak diffuse auroral arcs recovered by means of auroral tomography. The tomographic images are obtained from sets of scanning multi-channel photometer data obtained in February 1999 on the Kola Peninsula in Russia at three sites of a chain extending $226 \mathrm{~km}$ along the geomagnetic meridian. The 427.8- and 557.7-nm emissions of a 15-s time resolution observed within one hour during low geomagnetic activity are analyzed. We found that the intensity profile of an individual arc along the geomagnetic meridian has an inverted-V-shape. The luminosity maximum altitude decrease by $4-14 \mathrm{~km}$ at about $140 \mathrm{~km}$ distance in the south-north direction can be observed during two or more diffuse arcs. The parameters of the precipitating electron flux are obtained from an integral equation, which determines the best relationship between the $427.8-\mathrm{nm}$ intensity height profile and an arbitrary particle energy spectrum. A dimensionless function of the energy dissipation is used as the core in the integral equation. The estimated average energy of electron flux, which generated the isolated diffuse arc, is $1-2 \mathrm{keV}$ higher in the central part of the arc in comparison to values at its borders.
\end{abstract}

Keywords. Ionosphere (Active experiments; Auroral ionosphere; Particle precipitation)

\section{Introduction}

Direct satellite and rocket optical observations of aurora phenomena, which were performed for many years, have collected a lot of information about processes occurring in the upper atmosphere during auroral precipitation. Such measurements are quite expensive but they cannot provide data

Correspondence to: Zh. V. Dashkevich

(dashkevich@pgi.kolasc.net.ru) needed to perform comprehensive studies of the electron flux generating auroral forms in the ionosphere. Ground-based observations help to obtain the necessary knowledge on the spatial distribution of auroral emission intensity and characteristics of the electron flux.

However, very important problems, such as explaining the fine structure of electron flux parameters producing discrete auroral formations (arcs, belts and separate rays), structural and dynamic properties of precipitation in various auroral forms, can be solved by using indirect ground-based methods. One of them is an investigation of the spatial structural peculiarities in the aurora luminosity.

As a rule, the pioneer papers describing altitude luminosity intensity profiles use data obtained by photographic or photometric observations performed at two separated stations (Störmer, 1955; Romick and Belon, 1967). Then the height distributions of the volume luminosity intensity are recovered under the assumption that an aurora structure is a thin layer of a homogeneous luminosity across the auroral form. Therefore, such experiments can only result in the estimation of the lower border and the maximum.

Further development may be based on auroral tomography, if a set of images is recorded simultaneously from multiple locations. The tomographic inversion can be used to estimate the spatial distribution of the volume emission rate in a physical coordinate system. It allows one to avoid the approximations made in the case of two observational sites. If the region of interest is $80-500 \mathrm{~km}$ away, the optical tomography detectors must also be separated by comparable distances.

The works by McDade and Llewellyn (1991), Frey et al. (1996) are focused on the detailed description of the tomographic inversion algorithm, which is used for reconstructing altitude intensity profiles and based on both rocket photometric data and ground-based observations. Some examples of the obtained distributions can be found in these papers. An investigation of an auroral arc system excited by soft

Published by Copernicus GmbH on behalf of the European Geosciences Union. 


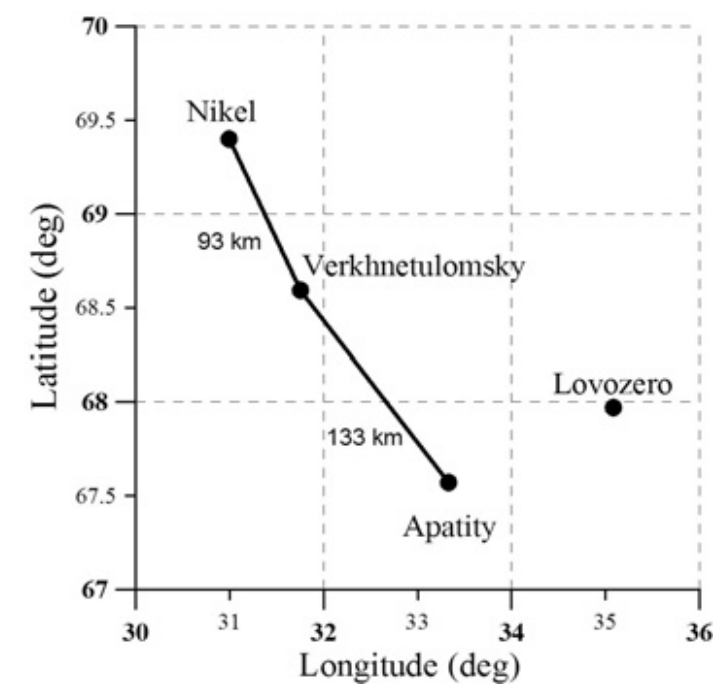

Fig. 1. The experiment geometry showing the locations of the scanning photometers.

electrons is made by using simultaneous rocket and groundbased measurements during the ARIES campaign (Vallance Jones et al., 1991; McDade et al., 1991). The authors studied altitude distributions of the volume emission rates at 470.9, $557.7,630.0$ and $844.6 \mathrm{~nm}$; a comparison of the tomographic images and direct rocket observations shows a reasonable agreement. It has been shown that the observed auroral arcs could be produced by an auroral electron flux of Maxwellian or Gaussian energy spectra with characteristic energies of the order of $3 \mathrm{keV}$. Frey et al. (1998) estimated some parameters of conductivity distributions by simultaneous electron density and spectral measurements made by the EISCAT radar and three CCD cameras. Some luminosity properties in the emissions of 557.7 and $630.0 \mathrm{~nm}$ for the Polar Cap arcs and mid-latitude auroras are investigated in the papers by Doe et al. (1997) and Semeter et al. (1999) by using the tomographic approach.

However, the features of the luminosity distribution just within an individual isolated auroral form cannot be found in the above papers. The objective of the present work is to study properties of both altitude and meridional distributions of the volume auroral emission rate, as well as to estimate the parameters of precipitating electron spectra corresponding to the intensity distributions. Our studies are based on the results of optical auroral tomography experiment, carried out by the Polar Geophysical Institute in the Kola Peninsula in 1999. The parameters under study are the average flux energy and the energy and particle fluxes. We use the tomographic algorithm based on stochastic inversion (Markkanen et al., 1995) modified for the inversion of optical data. For our analysis we selected time intervals when one or two weak diffuse arcs of the 557.7-nm emission with intensities less than $2.5 \mathrm{kR}$ can be clearly observed.
Table 1. Technical specifications of the photometers.

\begin{tabular}{|c|c|c|c|c|}
\hline \multicolumn{5}{|c|}{ Photometer specifications } \\
\hline Resolution & \multicolumn{4}{|c|}{$0.5-0.8^{\circ}$} \\
\hline Sensitivity & \multicolumn{4}{|c|}{$5-20 \mathrm{P}$} \\
\hline Dynamic range & \multicolumn{4}{|c|}{$3 \times 10^{4}$} \\
\hline Field-of-view & \multicolumn{4}{|c|}{$0-180^{\circ}$} \\
\hline Scan step & \multicolumn{4}{|c|}{$0.5^{\circ}$} \\
\hline All-sky scan time & \multicolumn{4}{|c|}{$15 \mathrm{~s}$} \\
\hline \multicolumn{5}{|c|}{ Filter characteristics } \\
\hline & \multicolumn{2}{|c|}{$4278 \AA ̊$} & \multicolumn{2}{|c|}{$5577 \AA ̊$} \\
\hline Apatity & 4280 & 18 & 5582 & 20 \\
\hline Verkhnetulomsky & 4275 & 18 & 5578 & 18 \\
\hline Nikel & $\begin{array}{l}4274 \\
\text { center } \AA\end{array}$ & $\begin{array}{l}17 \\
\text { width } \AA\end{array}$ & $\begin{array}{l}5580 \\
\text { center Å }\end{array}$ & $\begin{array}{l}19 \\
\text { width } \AA\end{array}$ \\
\hline
\end{tabular}

\section{Experiment description}

The brightness of the auroral emission was registered by three scanning photometers installed on a chain lying approximately along the magnetic meridian. The optical receivers were installed in Apatity, Verkhnetulomsky and Nikel, located from south to north, respectively. During the photometer observations, the all-sky camera images were also obtained at Lovozero Observatory, which is located close to the southern station. Figure 1 presents the experiment geometry. The main viewing line of the scanners between Apatity and Nikel has the azimuth of $323.82^{\circ}$, the viewing line of the scanner at Verkhnetulomsky was also parallel to the main viewing direction. This scanner location was about $8 \mathrm{~km}$ westward relative to the line between Apatity and Nikel. The instruments were directed along the operational azimuth with an accuracy of about $\pm 0.25^{\circ}$. The four-channels scanning photometers are described in Korotkov et al. (1997). Table 1 shows the technical specifications of the photometers. The scanning rate of the photometers is four scans per minute and the timing accuracy of the computers is about one second. The instruments are able to record four emissions, including 557.7 and $427.8 \mathrm{~nm}$.

The diffuse arcs of 557.7-nm brightness and approximately of $2.5 \mathrm{kR}$ are studied by using all three sets of experimental data. These arcs are well observed at wide viewing angles of all sites at the midnight hours. Figure 2 shows a $557.7-\mathrm{nm}$ brightness time history observed by the photometers. A magnetogram recorded at Lovozero Observatory is also included here. One can see from the magnetogram that the ionosphere is quiet on 11 February before 00:35 UT, but later, however, a storm occurred with its peak at about 01:00 UT. The hourly magnetic K-index on 10 February at 23:20 UT is close to 1 , but it is about 5 on 11 February at 01:00 UT when the breakup is the strongest. A detailed examination of the all-sky TV camera images also confirms that 

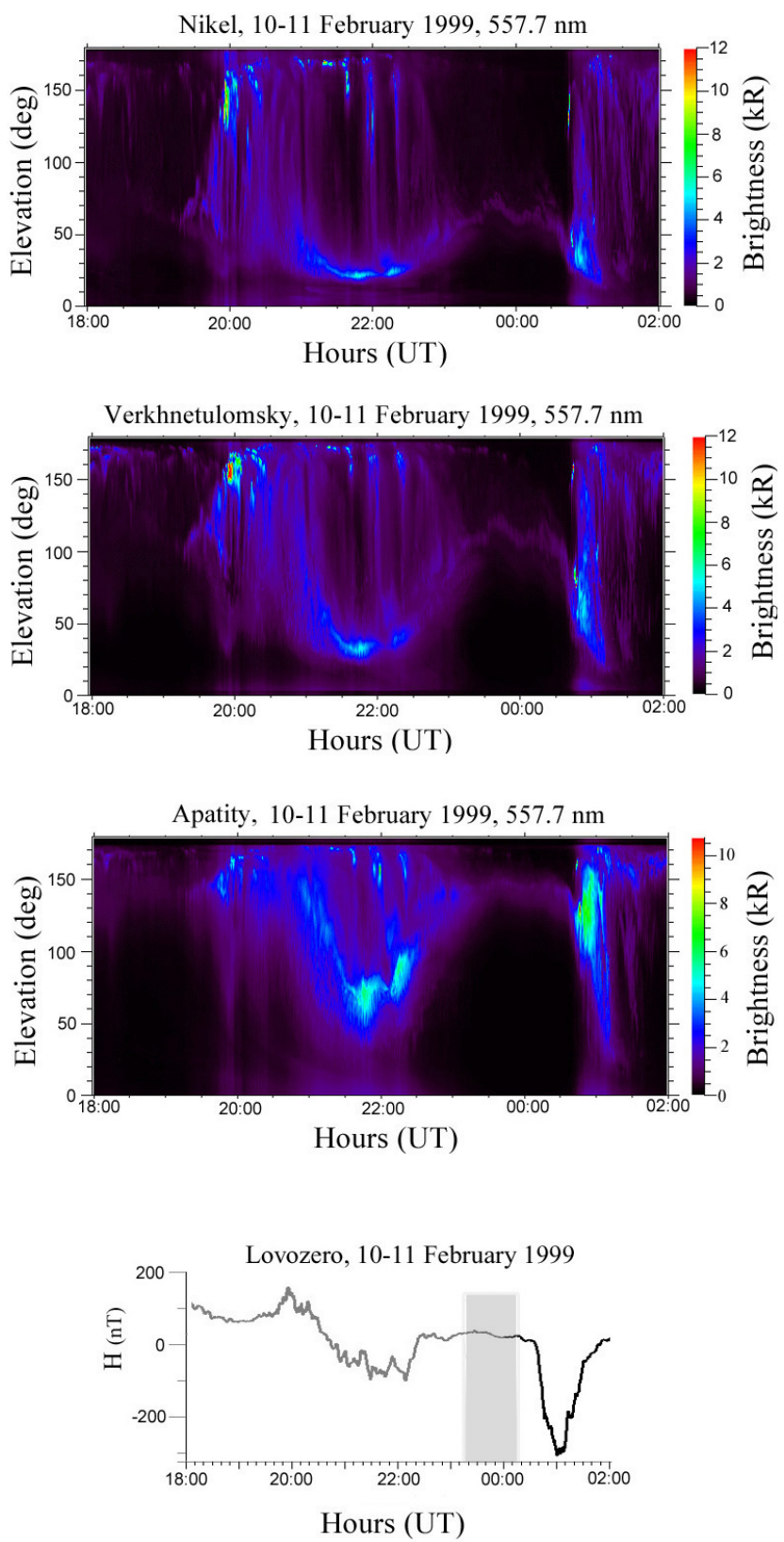

Fig. 2. The intensities of the emission $557.7 \mathrm{~nm}$ measured at three sites, as well as the magnetogram obtained at Lovozero Observatory on 10-11 February 1999. The gray bar shows the aurora observing period.

two stable arcs and a series of slightly moving arcs existed between 23:10-00:30 UT. We selected the following observational times for study at 23:17, 23:19, 23:21, 23:50, 00:01, and 00:15 UT. It is rather important to obtain correct estimates of the emission brightness from the experimental data, however, it seems to be quite difficult to estimate true background values by means of direct measurements. It might be acceptable that the background values correspond to the

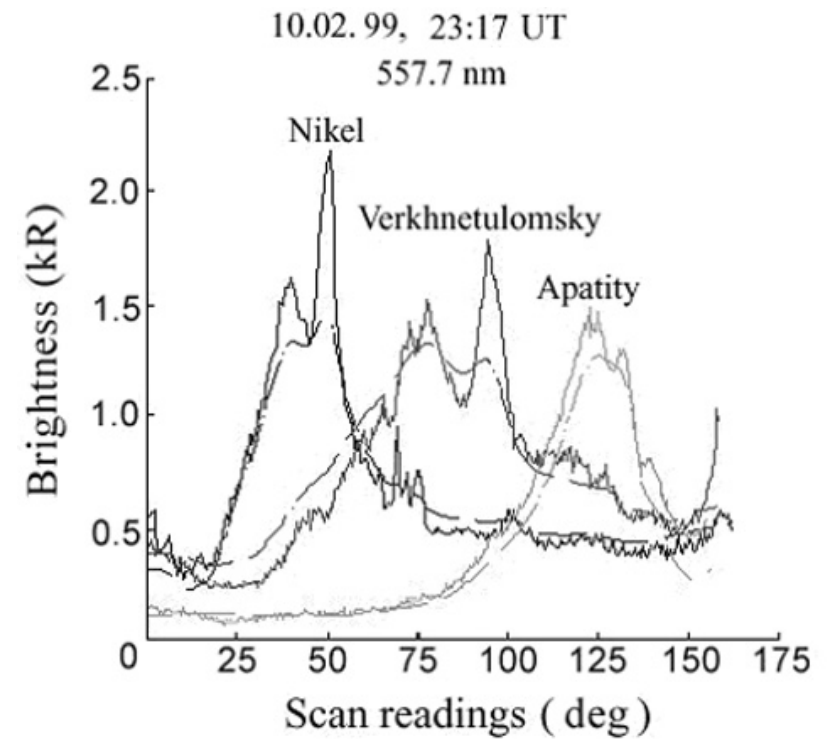

Fig. 3. Luminosity records obtained at the sites at the same time during quiet geomagnetic conditions. The measured records are shown as the solid lines, and the corresponding smoothed dashed lines represent the values obtained from the tomographic reconstructions.

minimum values of brightness recorded at times without any detectable aurora.

\section{Results}

3.1 Tomographic reconstruction of 427.8-nm and 557.7nm luminosities

The auroral tomography analysis is based on the Bayesian stochastic approach. The idea is to find the most probable values of the volume emission rate in the selected grid points. This approach has been successfully used in satellite tomography for reconstructing the electron density in the ionosphere during more than ten years (Makkanen et al., 1995; Kunitsyn and Tereshchenko, 2003). We applied it here for reconstruction of the spatial distributions of auroral emissions. The inversion programs used in satellite tomography have been modified for reconstructing the volume emission rate of aurora, as in Nygrén et al. (1996). In our auroral tomography analysis the pixel size of the reconstruction grid is 2.5 by $2.5 \mathrm{~km}$, while in the satellite phase difference tomography the grid cell size is usually about 30 by $30 \mathrm{~km}$ or slightly less.

Bigaussian regularization is used as an initial guess in obtaining emission rate images. A usual criterion of estimating reconstruction errors is the values and number of negatives within the grid, which do not exist in reality. Another one is a comparison of initial raw luminosity records and those found from reconstructed images. Discrepancies 

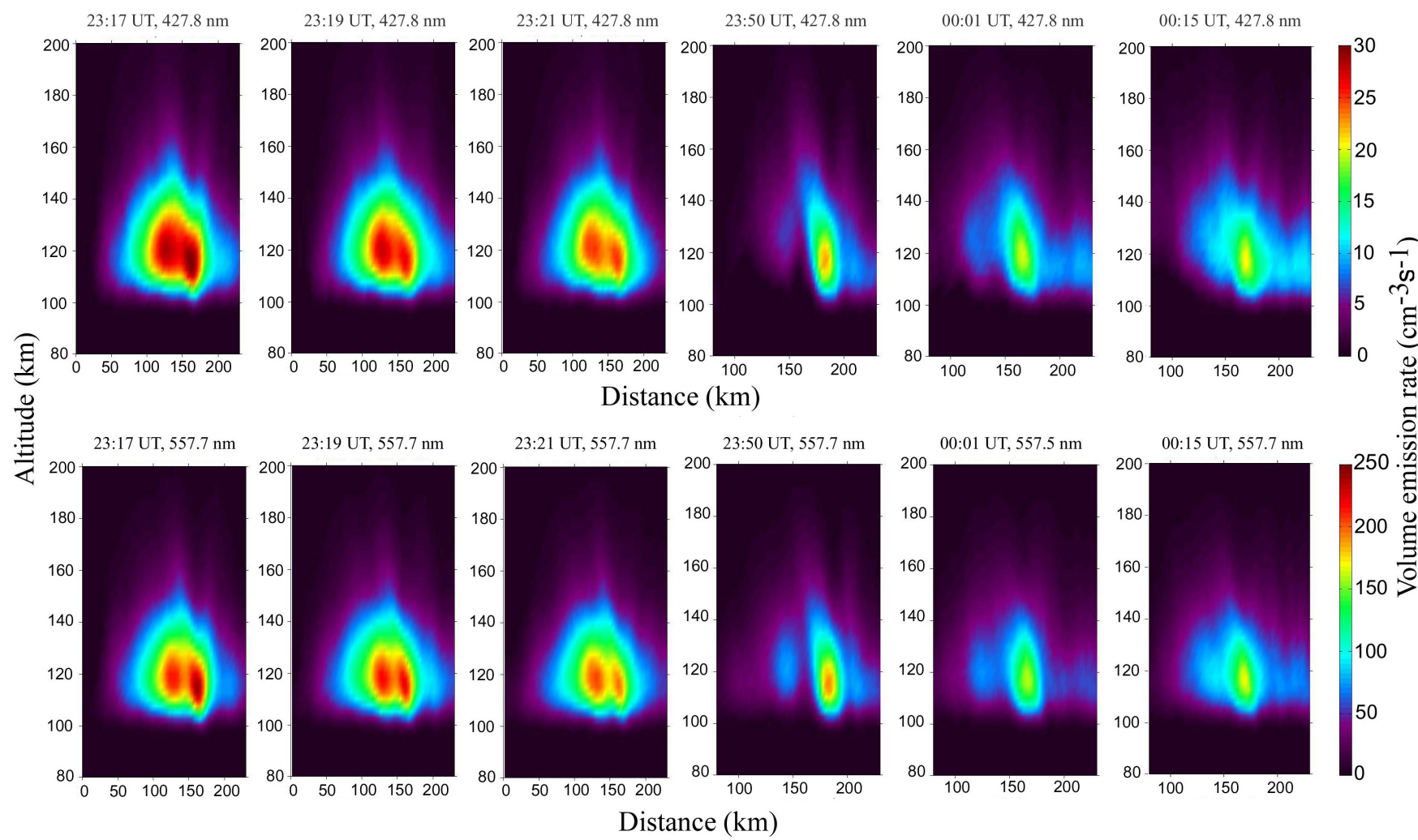

Fig. 4. Tomographic reconstructions of the 427.8- and 557.7-nm volume emission rates on 10-11 February 1999.

between the curves also depend on data quality, stability of investigated processes and some other reasons. The dashed curves in Fig. 3 are obtained from the reconstructions, and its smoothness depends on a grid cell size. The reconstructed curves shown in the figure satisfy the above criteria. They were selected after more than a dozen inversion procedures with different regularization profiles. The preliminary reconstructed images of the auroral volume rate distribution can be found in Kunitsyn and Tereshchenko (2003) and Efstafiev et al. (2001).

Figure 4 displays the tomographic images above the chain of the optical instruments on 10-11 February 1999 during quiet and disturbed ionosphere conditions at 23:17, 23:19, 23:21, 23:50, 00:01, and 00:15 UT. The X-axis shows the distance from Apatity. One can see that reconstructions display main morphological peculiarities of the luminosity structures seen well in the all-sky camera frames, for example, the two closely located arcs in the meridional plane along the chain at 23:17-23:19 UT on 10 February. Another distinctive feature of the images is an elongation of luminous structures approximately along the geomagnetic field line, which is inclined by about $13^{\circ}$ to the south from the vertical in this region. This peculiarity of the reconstructed images is an additional confirmation of the consistency of our results conforming to conventional concepts of the electron beam motion in the atmosphere.
Finally, we show a tendency for a decrease in altitudes of both the lower edge of the entire auroral structures and the maximum luminosity inside the auroral forms in the southto-north direction. This, in turn, is a confirmation that the hardness of the precipitated electron beams is increasing northward.

\subsection{Volume emission rate profiles and experimental inten- sities ratio}

The reconstructions of auroral luminosity allow us to estimate the height profiles of auroral emission intensities at any position of the aurora meridional cross sections. An example of reconstructed height profiles for the volume emission rates of 427.8 and $557.7 \mathrm{~nm}$ is displayed in Fig. 5a. The maximum luminosity positions are determined from distributions of the auroral intensities shown in the previous Fig. 4. The profiles are shown at the positions of the maximum luminosity at various observation times. As was mentioned above, the distances here originate from the reference station of Apatity. When the two arcs at 23:19 UT were observed, the distance of the southern arc luminosity maximum from Apatity is $130 \mathrm{~km}$ and the northern arc distance is $165 \mathrm{~km}$. It can be seen in Fig. 5a that at the same time the southern arc at 23:19 UT is located higher than the northern arc. The effect of decreasing the altitude of the maximum luminosity 
in the south-to-north direction is shown in Fig. 5b. Here the volume emission rate profiles of $427.8 \mathrm{~nm}$ at 23:21, obtained at various distances from Apatity, are also shown. It is clear that the altitude of the volume emission rate peak decreases when moving from Apatity.

The height profiles of the 557.7-nm and 427.8-nm intensities ratio are presented in Fig. 5c. Here we also show some experimental results obtained by different researchers for similar types of aurora (Feldman, 1978; Vallance Jones, 1979; Sharp et al., 1979; Deans et al., 1978; McDade et al., 1985; Stelle et al., 1990). Our intensity ratios are in the same interval as reported in the above papers. The ratio $I_{557.7} / I_{427.8}$ values are $6-9$ at heights from 90 to $120 \mathrm{~km}$, which correspond to the aurora luminosity maximum.

3.3 Meridional features of aurora emission intensity characteristics and parameters of the precipitating electron flux

In order to estimate the parameters of auroral electron flux, we use the technique described in detail in Appendix A. First, we have estimated the spectra of primary electron precipitating during the considered events on 10-11 February 1999. The electron spectra were reconstructed using the height profile of the 427.8-nm volume emission rates obtained from the tomographic reconstructions.

Let us consider how the following parameters act inside an auroral formation. $I_{427.8}$ is the $427.8-\mathrm{nm}$ emission intensity integrated over a vertical column, $I_{557.7} / I_{427.8}$ is the intensity ratio, $H_{\max }$ is the height of the $427.8-\mathrm{nm}$ volume emission rate peak $\left(I_{\max }\right), F_{e}$ is the energy flux and $E_{e v}$ is the mean energy of the precipitating electron flux.

Figure 6 presents some examples of change in these characteristics along the meridional direction inside the auroral formation during the period between 23:17 and 00:15 UT, covering the extended quiet phase of the aurora. Notice that at 23:17, 23:19, 23:21 UT two arcs are observed and only one auroral arc is observed at 00:01 and 00:15 UT. The altitude of the volume emission rate peak and the 427.8-nm emission intensity were estimated from tomography reconstructions. There is a tendency of decreasing $I_{\max }$ in the south-north direction by about $4-14 \mathrm{~km}$, which corresponds to increasing the mean electron energy along the meridian by $1-2 \mathrm{keV}$. In most cases, the intensity ratio $I_{557.7} / I_{427.8}$ varies between 7 and 5, thus displaying a tendency of decreasing northward. At the same time, it was expected that the energy flux should have a maximum near the center of the luminosity region and a decrease toward the arc edges, as well as a correlation with the 427.8-nm emission intensity.

Figure 7 shows a common tendency of variations of both the altitude of the 427.8-nm volume emission rate peak and the mean energy of the precipitated flux along the meridian. The events were grouped at the time intervals of 23:1723:21 UT and 23:50-00:15 UT. It can be seen that the altitude of the volume emission rate peak decreases in the south-north
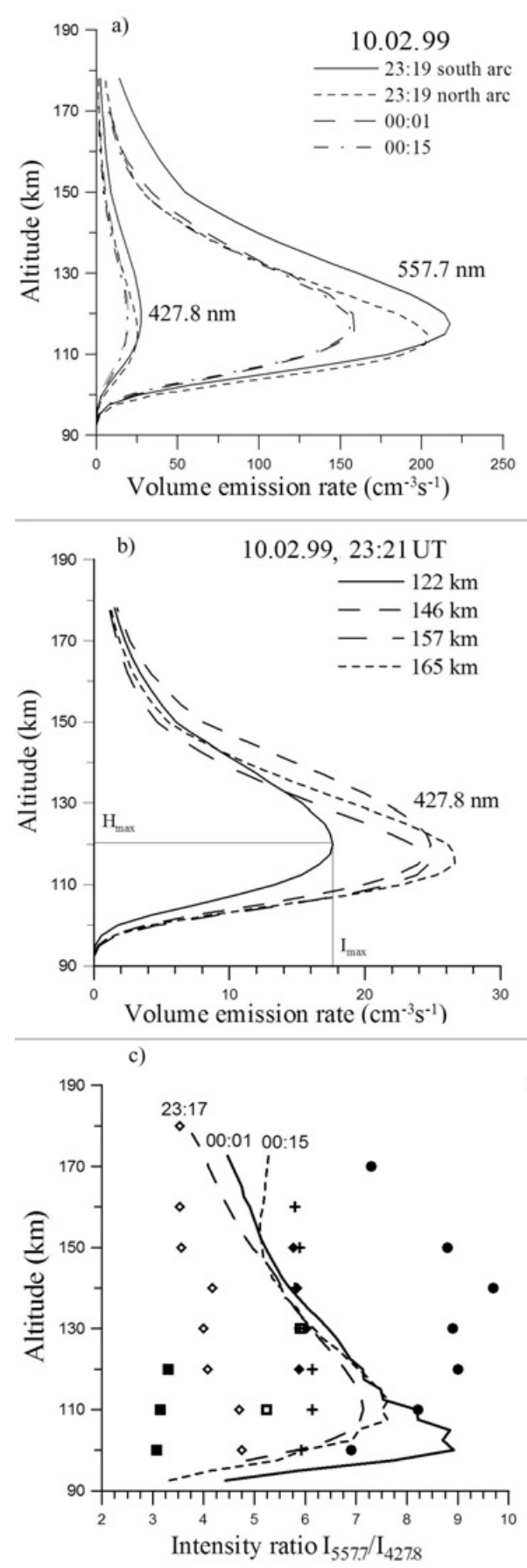

Fig. 5. (a), (b): Volume emission rates of 427.8 and $557.7 \mathrm{~nm}$ obtained during various observational conditions; (c): Intensity ratio $I_{557.7} / I_{427.8}$. Lines mark the results, obtained trom tomographic reconstructions, crosses mark the results by Feldman (1978), solid rhomb - Vallance Jones (1979), open rhomb - Sharp et al. (1979), circle - Deans et al. (1978), solid square - McDade et al. (1985), open square - Stelle et al. (1990). 


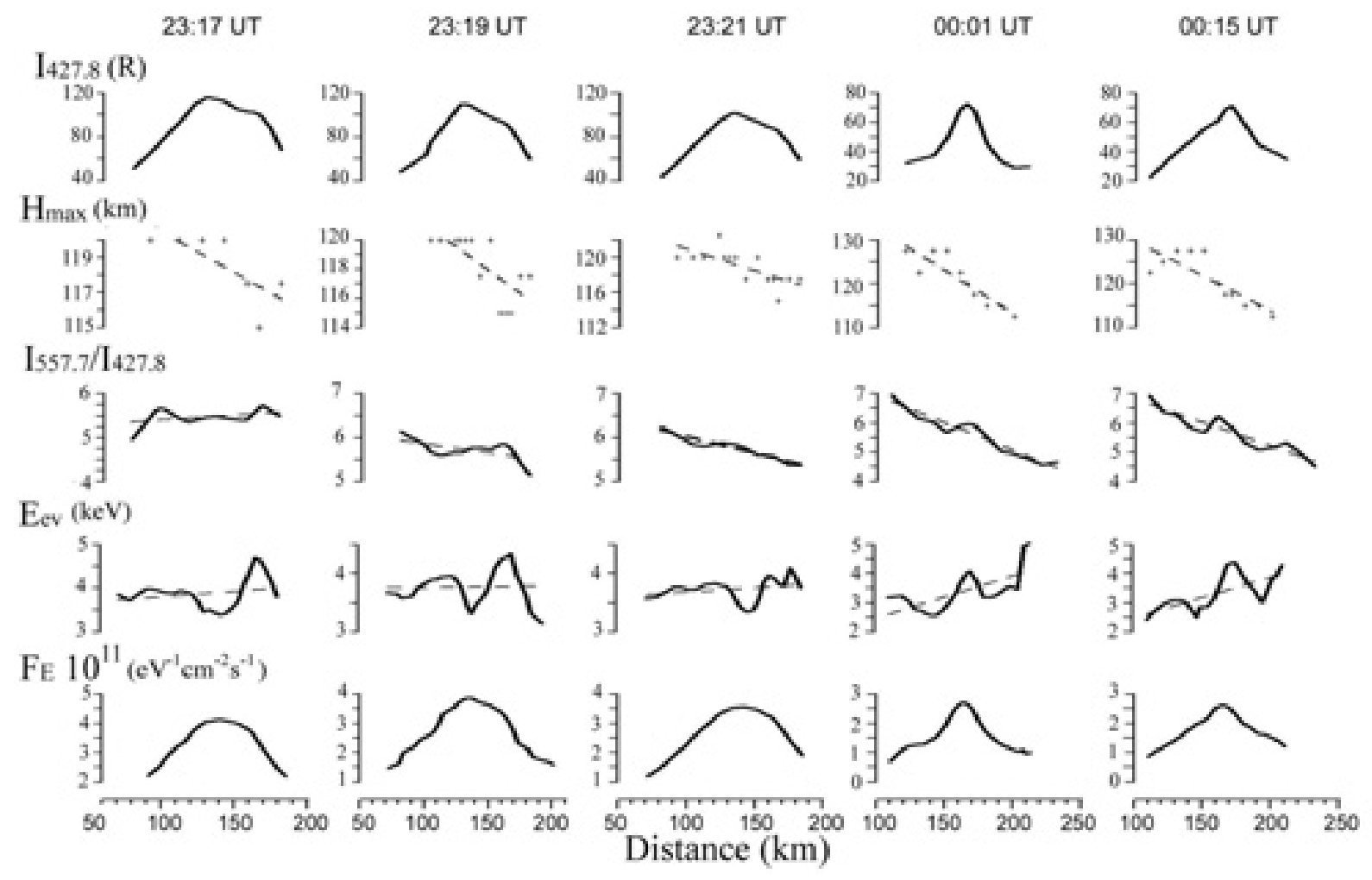

Fig. 6. Meridional variations of different auroral emission intensities and parameters of the precipitating electron flux inside the entire auroral formation.
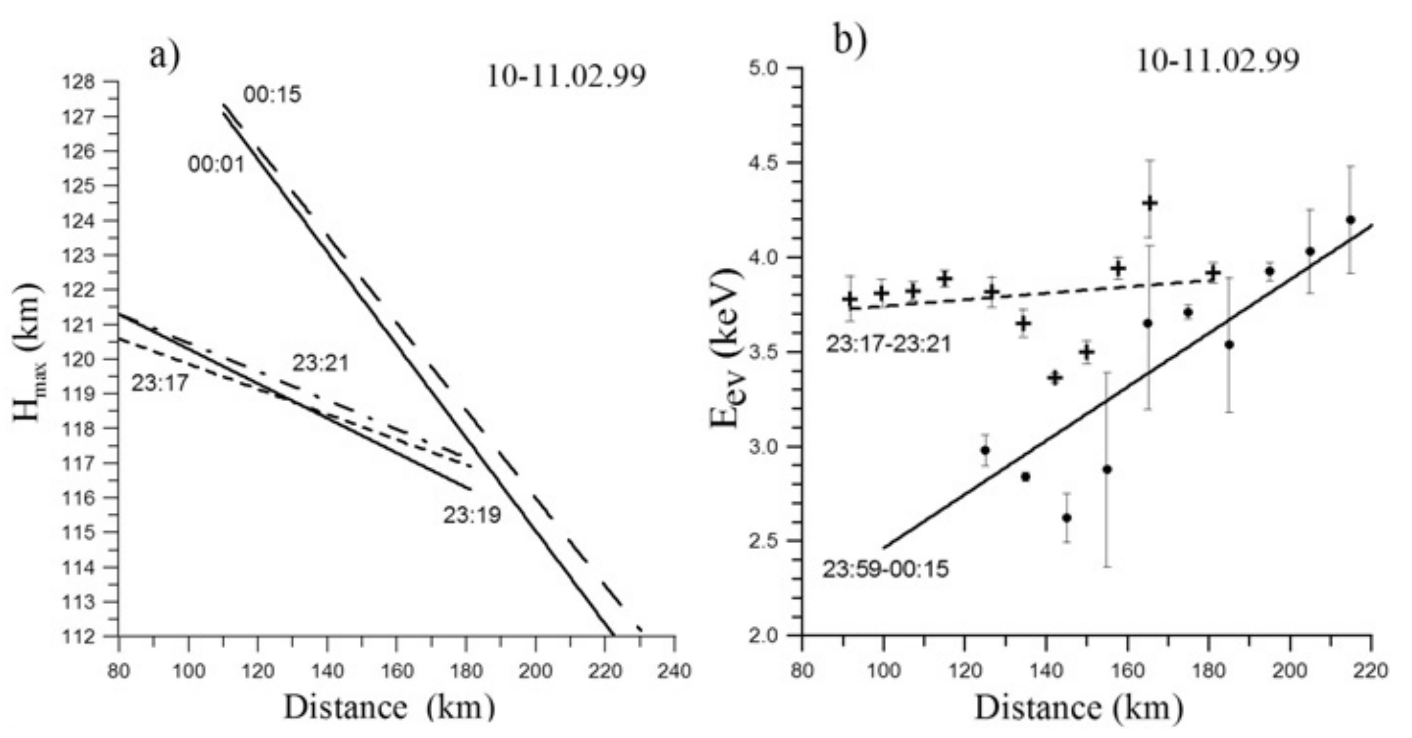

Fig. 7. Variations of the altitude of peak of the 427.8-nm volume emission rate along the meridian (a) and the mean energy of the precipitating electrons (b) at various times in a wide observational sector. The mean electron energy is averaged individually during both events when we observed only one arc at 23:17-23:21 UT or two arcs at 23:50-00:15 UT. The vertical bars indicate the confidence interval.

direction along the geomagnetic meridian while the mean electron energy increases.
The next step is to study the small-scale structure of the distribution of the luminosity characteristics and electron flux parameters. Figure 8 (the upper panel) represents the 


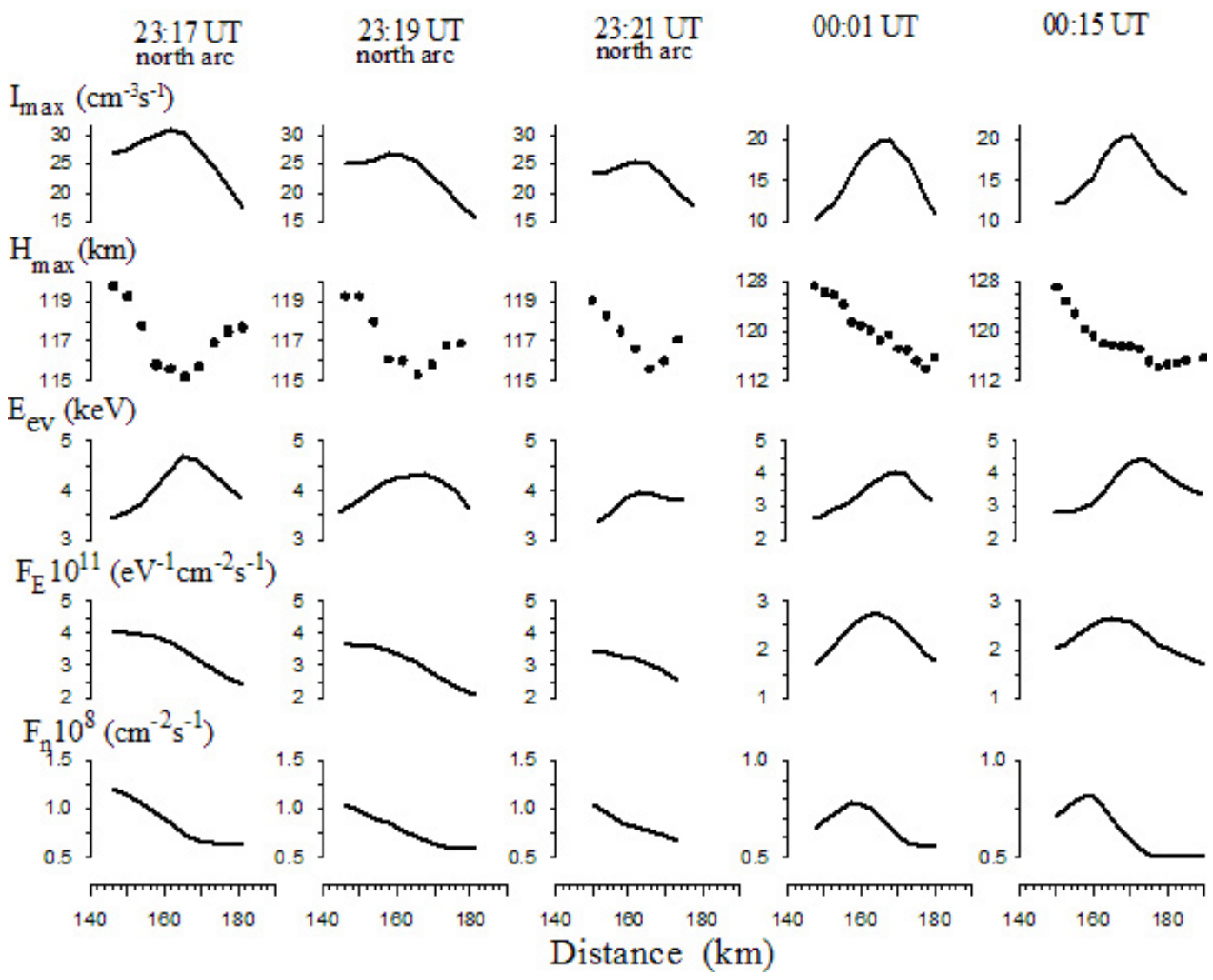

Fig. 8. Meridional behavior of auroral emission intensity characteristics and parameters of the precipitating electron flux inside the individual arcs.

curves describing features of the 427.8-nm volume emission rate peak $I_{\max }$ and the height of this peak $H_{\max }$. Only the northern arc can be seen at 23:17-23:21 UT. We see from the figure that the variations in the volume emission rate peak $I_{\max }$ are quite opposite to the variations in the altitude of this peak $H_{\max }$. Because these parameters vary across the arc, we can first conclude that the hardness of the electron beam inside the arc close to its center is maximal, which is caused, in turn, by the meridional distribution of the mean energy within the electron flux which forms separate auroral arcs. We can see the variations of the electron flux parameters across the arcs: the energy $F_{E}$, the mean energy of the precipitating electron flux $E_{e v}$ and the particle flux $F_{n}$. We can also clearly see the inverted V-structure in the values of the mean electron energy across the arc for all cases in our study. However, the variation features of the energy and particle fluxes differ at different times. We see a similar $\mathrm{V}$-structure in both the energy and in the particle flux values across the arc at 00:01 and 00:15 UT when only one arc is observed. During appearances of two arcs at 23:17, 23:19 and 23:21 UT the inverted V-structure is observed only in the mean energy of the electrons while the energy and electron fluxes smoothly decrease from the south to the north.
The results obtained show that if only one arc exists, then all the parameters of the precipitating electrons (the mean energy, energy flux and particle flux) have the inverted Vforms along this auroral arc. If several arcs exist, the inverted $\mathrm{V}$ - structure is observed in the mean energy of electrons only. The energy flux and the particle flux are smoothly decreasing towards the north.

\section{Conclusions}

The experimental results obtained by the optical tomography have been described in this paper. Three scanning photometers of four channels were installed along the meridional chain observing 427.8 and $557.7 \mathrm{~nm}$ auroral emissions. Two-dimensional images of a stable diffuse arc observed on 10-11 February 1999 have been obtained in the form of 557.7- and 427.8-nm intensity distributions. The values of ratios $I_{557.7} / I_{427.8}$ lie between 6 and 9 at heights of about $90-120 \mathrm{~km}$, which are in agreement with earlier experimental works. The spectra of electrons precipitating during the selected events have been calculated. Several peculiarities of the meridional variations of both the precipitating 

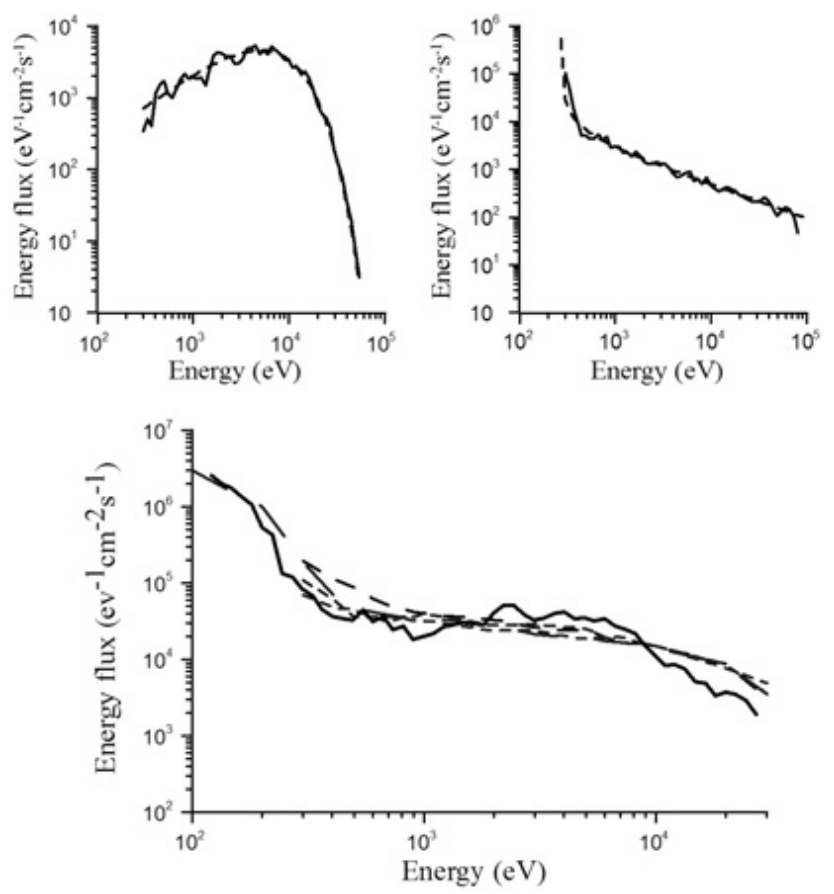

Fig. 9. The upper panel: examples of reconstruction of energy spectra. The lower panel: all dashed lines illustrate the energy spectra measured by the AE-C satellite on 21 March 1974 (Rees et al., 1977), the solid line indicates the reconstructed energy spectrum using experimental high profile of $381.4 \mathrm{~nm}$ intensity (Sharp et al., 1979).

electron parameters and auroral emission intensity characteristics have been revealed, namely:

1. A tendency for a decrease in the altitude of the emission intensity peak in the south-north direction can be revealed inside the entire auroral structures. It was found that height variations of the emission intensity peak could be about $4-14 \mathrm{~km}$ at about $140-\mathrm{km}$ distance. We can also see a tendency for an increase in the mean electron energy in the same direction up to $2 \mathrm{keV}$.

2. A typical feature inside an individual isolated arc is an inverted V-structure observed in the mean electron energy along the meridian, as well as corresponding Vshaped changes in the altitude of the emission intensity peak across the arc.

\section{Appendix A}

\section{Reconstruction of the initial energy spectrum of the precipitating electron flux}

The substance of this method is in estimating the initial energy spectrum of the electron flux by means of experimen- tally obtained altitudinal intensity distributions of optically permitted auroral emissions.

The volume emission rate $I_{\lambda}(h)$ at the altitude $h$ can be defined by the following expression:

$I(\lambda, h)=n(h) \cdot A_{\lambda}$,

where $n(h)$ is the concentration of the excited particles, $A_{\lambda}$ is the Einstein coefficient, and $\lambda$ is the wavelength.

The concentration of excited particles which is not subject to processes of collisional quenching, under steady-state conditions can be found as follows:

$Q(h)-n(h) \cdot \sum A_{i}=0$,

where $Q(h)$ is the excitation rate by the direct electron impact, $\Sigma A_{i}$ is the sum of the Einstein coefficients of all possible optical transitions for the current state.

The $Q(h)$-expression can be found, for example, in the paper by Sergienko and Ivanov (1993). It is based on the exact solution of the electron transfer problem by the Monte-Carlo method. In this paper the relation between the initial spectrum of auroral electrons at the upper boundary of the ionosphere $F(E)$ and the excitation rate of the atmospheric gas $Q(h)$ is solved by using the function of the electron energy dissipation.

The results derived in the paper by Sergienko and Ivanov (1993) give the following expression of the volume emission rate:

$I(\lambda, h)=\frac{A_{\lambda}}{\sum A_{i}} \int_{E} K(h, E) \cdot F(E) d E$,

where

$K(h, E)=\frac{\rho(h) P_{j}(h)}{\varepsilon_{j}^{i}(h)} \frac{E \lambda(E, \chi)}{R(E)}(1-A l b(E))$,

where $\rho(h)$ is the atmosphere density at the height $h$ in $\mathrm{g} / \mathrm{cm}^{3}$, $\mathrm{P}_{j}(h)$ is the dimensionless multiplier corresponding to the $j$ th kind of the gas, $\lambda(E, \chi)$ is the dimensionless dissipation function, $\chi$ is the distance from the upper boundary of the ionosphere to the altitude $h$, expressed in units of the passable mass in $\mathrm{cm}^{2} / \mathrm{g}, \varepsilon_{j}^{i}(h)$ is the energy cost of the gas with the $i$-th state and $j$-th kind in $\mathrm{eV}, R(E)$ is the average range of the electron flux from the upper boundary of the ionosphere in $\mathrm{g} / \mathrm{cm}^{2}, A l b(E)$ is the albedo-flux.

So, in order to determine the initial electron flux from the altitude profile of volume emission, it is necessary to solve an integral equation. Applying experimental values of $I(\lambda, h)$ and expressing the integral as a sum by the trapezoid method, we obtain:

$$
\begin{aligned}
I(\lambda, h)= & \frac{A_{\lambda}}{\sum_{i} A_{i}}\left(\frac{K\left(h, E_{1}\right) F_{1} \Delta E_{1}}{2}+\frac{K\left(h, E_{M}\right) F_{M} \Delta E_{M}}{2}\right. \\
& \left.+\sum_{k=2}^{M-1} \frac{K\left(h, E_{k}\right) F_{k}\left(\Delta E_{k}-\Delta E_{k-1}\right)}{2}\right)
\end{aligned}
$$


Then the problem reduces to the determination of $F_{k}$ values by the least-squares method.

We have tested the present method by two arbitrarily defined initial distributions of the auroral electrons, such as $F(E)=C \cdot e^{-E / 5000}$ and $F(E)=C \cdot(E-270)^{-0.7}$. The altitudinal profiles of the 427.8-nm emission volume rates of $1 \mathrm{NG}$ short-lived molecular nitrogen ions are estimated using these spectra. Next we calculate the spectra using these profiles and the MSIS-86 atmospheric model. The results of testing are shown in Fig. 9, where the defined and reconstructed electron energy spectra are presented. A qualitative comparison of the defined and estimated spectra leads to a conclusion that our technique of the electron spectra reconstructing might give quite good results.

One more examination of this method was made by using experimental data obtained during the coordinated rocketsatellite campaign (Rees et al., 1977; Sharp et al., 1979). The experimental and reconstructed spectra are shown at the bottom of Fig. 9. It is reconstructed using the experimental height profile of the 391.4-nm intensity. An assumption is taken into account that the pitch-angle electron distribution is isotropic, which is acceptable for quiet forms of aurora. The values of the neutral gases density were borrowed from the above rocket-satellite experiment. A conclusion can be made that the experimental spectra and the ones estimated here are in a good agreement.

Acknowledgements. The authors are grateful to S. V. Leontyev, A. V. Roldugin, B. G. Korotkov for their help in carrying out the experiment as well as to E. D. Tereshchenko, PGI Director, for his valuable comments and to Yu. V. Fedorenko for proofreading of the text.

Topical Editor M. Pinnock thanks two referees for their help in evaluating this paper.

\section{References}

Deans, A. J. and Shepherd, G. G.: Rocket measurements of oxygen and nitrogen emissions in the aurora, Planet. Space Sci., 26(4), 319-333, 1978.

Doe, A. R., Kelly, J. D., Semeter, J. L., and Steele, D. P.: Tomographic reconstruction of $630.0 \mathrm{~nm}$ emission structure for a polar cap arc, Geophys. Res. Lett., 4(9), 1119-1122, 1997.

Evstafiev, O. V., Dashkevich, Zh. V., Khudukon, B. Z., and Tereshchenko, E. D.: Combined satellite and auroral tomographic measurements over a receiver chain in Russia, Proceedings of the International Beacon Satellite Symposium 2001, Boston, 3-6 June 2001.

Feldman, P. D.: Auroral excitation of optical emissions of atomic and molecular oxygen, J. Geophys. Res., 83(A6), 2511-2516, 1978.

Frey, S., Frey, H. U., Carr, D. J., Bauer, O. H., and Haerendel, G.: Auroral emission profiles extracted from three-dimensionally reconstructed arcs, J. Geophys. Res., 101(A10), 21 731-21 741, 1996.

Frey, H. U., Frey, S., Lanchester, B. S., and Kosch, M.: Optical tomography of the aurora and EISCAT, Ann. Geophys., 16(10), 1332-1342, 1998.
Korotkov, V. G., Kozelov, B. V., and Leontyev, S. V.: Auroral mechanically scanning photometer, in: Devices and methods of geophysical experiment, Kola Science Cente of Russian Academy of Science, Murmansk, 15-20, 1997.

Kunitsyn, V. E. and Tereshchenko, E. D.: Ionospheric tomography, Springer-Verlag, Berlin, Heidelberg, 259 p, 2003.

Makkanen, M., Lehtinen, M., Nygrén, T., Pirttila, J., Henelius, P., Vilenius, E., Tereshcenko, E. D., and Khudukon, B. Z.: Bayesian approach to satellite radiotomography with applications in the Scandinavian sector, Ann. Geophys., 13, 1277-1287, 1995, http://www.ann-geophys.net/13/1277/1995/.

McDade, I. C., Llewelyn, E. J., and Solheim, B. H.: A rocket measurement of $\mathrm{O}\left({ }^{1} \mathrm{~S}\right)$ and $\mathrm{N}_{2}^{+}$emissions in a pulsating aurora, Can. J. Phys., 63, 983-987, 1985.

McDade, I. C., Lloyd, N. D., and Llewellyn, E. J.: A rocket tomography measurement of the $\mathrm{N}_{2}^{+} 3914 \AA$ emission rates within an auroral arc, Planet. Space Sci., 39(6), 895-906, 1991.

McDade, I. C. and Llewelyn, E. J.: Inversion techniques for recovering two-dimensional distributions of auroral emission rates from tomographic rocket photometer measurements, Can. J. Phys., 69, 1059-1068, 1991.

Nygrén, T., Makkanen, M., Lehtinen, M., and Kaila, K.: Application of stochastic inversion in auroral tomography, Ann. Geophys., 14, 1124-1133, 1996, http://www.ann-geophys.net/14/1124/1996/.

Rees, M. H., Stewart, A. I., Sharp, W. E., Hays, P. B., Hoffman, R. A., Brace, L. H., Doering, J. P., and Peterson, W. K.: Coordinated rocket and satellite measurements of an auroral event, 1, Satellite observation and analysis, J. Geophys. Res., 82(16), 2250-2261, 1977.

Romick, G. J. and Belon, A. E.: The spatial variation of auroral luminosity - II Determination of volume emission rate profiles, Planet. Space. Sci., 15, 1695-1716, 1967.

Semeter, J., Mendillo, M., and Baumgardner, J.: Multispectral tomographic imaging of the midlatitude aurora, J. Geophys. Res., 104(A12), 24 565-24 585, 1999.

Sergienko, T. I. and Ivanov, V. E.: A new approach to calculate the excitation of atmospheric gases by auroral electron impact, Ann. Geophys., 11, 717-724, 1993,

http://www.ann-geophys.net/11/717/1993/.

Sharp, W. E., Rees, M. N., and Stewart, A. I.: Coordinated rocket and satellite measurements of on auroral event. 2. The rocket observations and analysis, J. Geophys. Res., 84(A5), 1977-1984, 1979.

Stelle, D. P. and McEwen, D. J.: Electron auroral excitation efficiencies and intensity ratios, J.Geophys. Res., 95(A7), 1032110336, 1990.

Störmer, C.: The PolarAurorae, Clarendon Press, Oxford, 1955.

Vallance Jones, A.: Measurements of the optical emission height profiles of medium intensity aurora, Planet. Space Sci., 27(3), 307-312, 1979.

Vallance Jones, A., Gattinger, R. L., Creutzberg, F., Harris, F. R., McNamara, A. G., Llewellyn, E. J., Lummerzheim, D., Rees, M. N., McDade, I. C., and Margot, J.: The ARIES auroral modelling camping: characterization and modeling of evening auroral arc observed from a rocket and a ground-based line of meridian scanners, Planet. Space Sci., 39(12), 1677-1705, 1991. 